\title{
Epigenomic landscape of enhancer elements during Hydra head organizer formation
}

\author{
Puli Chandramouli Reddy ${ }^{\dagger}$ (D), Akhila Gungi ${ }^{\dagger}$ (D), Suyog Ubhe and Sanjeev Galande* (D)
}

\begin{abstract}
Background: Axis patterning during development is accompanied by large-scale gene expression changes. These are brought about by changes in the histone modifications leading to dynamic alterations in chromatin architecture. The cis regulatory DNA elements also play an important role towards modulating gene expression in a contextdependent manner. Hydra belongs to the phylum Cnidaria where the first asymmetry in the body plan was observed and the oral-aboral axis originated. Wnt signaling has been shown to determine the head organizer function in the basal metazoan Hydra.
\end{abstract}

Results: To gain insights into the evolution of cis regulatory elements and associated chromatin signatures, we ectopically activated the Wnt signaling pathway in Hydra and monitored the genome-wide alterations in key histone modifications. Motif analysis of putative intergenic enhancer elements from Hydra revealed the conservation of bilaterian cis regulatory elements that play critical roles in development. Differentially regulated enhancer elements were identified upon ectopic activation of Wnt signaling and found to regulate many head organizer specific genes. Enhancer activity of many of the identified cis regulatory elements was confirmed by luciferase reporter assay. Quantitative chromatin immunoprecipitation analysis upon activation of Wnt signaling further confirmed the enrichment of H3K27ac on the enhancer elements of Hv_Wnt5a, Hv_Wnt11 and head organizer genes Hv_Bra1, CnGsC and Hv_Pitx1. Additionally, perturbation of the putative H3K27me3 eraser activity using a specific inhibitor affected the ectopic activation of Wnt signaling indicating the importance of the dynamic changes in the H3K27 modifications towards regulation of the genes involved in the head organizer activity.

Conclusions: The activation-associated histone marks H3K4me3, H3K27ac and H3K9ac mark chromatin in a similar manner as seen in bilaterians. We identified intergenic cis regulatory elements which harbor sites for key transcription factors involved in developmental processes. Differentially regulated enhancers exhibited motifs for many zinc-finger, T-box and ETS related TFs whose homologs have a head specific expression in Hydra and could be a part of the pioneer TF network in the patterning of the head. The ability to differentially modify the H3K27 residue is critical for the patterning of Hydra axis revealing a dynamic acetylation/methylation switch to regulate gene expression and chromatin architecture.

Keywords: Hydra, Head organizer, Enhancer, Histone modifications, Cis regulatory elements

*Correspondence: sanjeev@iiserpune.ac.in

${ }^{\dagger}$ Puli Chandramouli Reddy and Akhila Gungi contributed equally to this work

Centre of Excellence in Epigenetics, Department of Biology, Indian Institute of Science Education and Research, Dr. Homi Bhabha Road, Pune 411008, India

\section{Background}

During the development of an organism, the morphology is determined through a series of patterning events discretizing the body via multiple axes such as the anterior/ posterior, dorsal/ventral and left/right. The break in the symmetry of the animal Bauplan was the consequence of axis generation. Elegant studies in the past have shown 
that this is due to molecular polarization via distribution of morphogens into distinct territories creating localized patterning $[1,2]$. Highly conserved molecules have been identified as the regulators of the animal body plan which include the Hox genes. These homeotic proteins ensure proper patterning and development of an organism and the presence of a cluster of Hox genes was thought to be a bilaterian invention [3] The identification and characterization of Hox genes in cnidarians and ctenophores suggested that the zootype hypothesis might not be true [4-11]. Further, mechanisms upstream of the Hox patterning system were identified and the $\mathrm{Wnt} / \beta$-catenin signaling pathway was shown to play a critical role in regulating Hox gene expression. Across Metazoa, the $\mathrm{Wnt} / \beta$-catenin signaling pathway is a part of the posterior patterning during development [12]. Additionally, its function upstream of the Hox genes led to postulations that Wnt might be the major player in the primary axis formation $[10,13]$.

The origin of a defined body axis in the animal kingdom occurred in the phylum Cnidaria that exhibit an oral-aboral axis. Studies in cnidarians and ctenophores have established that unlike in higher phyla, Hox genes do not display polarized localization across the axis [10, 14]. In Hydra and other cnidarians, the canonical Wnt/ $\beta$-catenin signaling pathway is known to form the head organizer and recent evidence suggests that it could play an initiating role in the formation of the foot as well [1518]. This makes $H y d r a$ an ideal model system to study the process of axis patterning where the primary longitudinal axis is the oral-aboral axis. We have identified the downstream molecular players of the Wnt/ $\beta$-catenin signaling pathway which resulted in the identification of multiple homeobox transcription factors that play a critical role in Hydra axis formation [19].

Regulation of gene expression is orchestrated by multiple short DNA sequences called cis regulatory elements that include promoters and enhancers. While promoters are the more proximal regulatory regions and surround the transcription start sites (TSS) of gene bodies, enhancers are distal elements that are located from few $\mathrm{Kb}$ to $1 \mathrm{Mb}$ away from the gene body [20]. In genomewide studies, the combinatorial occupancy of histone modifications is typically used as predictive markers for enhancer identification [21-23]. The typically promoterassociated H3K4me3 modification has also been used as a signature of active enhancers in T-cells [24]. Enhancers can be categorized as active and poised enhancers. Active enhancers predominantly exhibit the H3K4me1 and H3K27ac modifications, whereas poised enhancers possess the H3K4me1 and H3K27me3 modifications [25]. Additionally, acetylation of $\mathrm{H} 3$ by $\mathrm{p} 300$ and related acetyltransferases on intergenic regions of the genome has been observed at enhancers [26, 27]. Enhancers and promoters can also be discriminated based on the type of RNAs produced from both these regulatory elements. Though both might have bidirectional transcription, enhancers typically produce fewer, shorter and less stable eRNAs restricted to the nucleus whereas promoters usually produce mRNAs that are highly stable, multiexonic, abundant and translated into proteins [28]. Enhancers and promoters also exhibit few sequence-based similarities in terms of transcription factor binding motifs/sites (TFBS). Presence of transcription factor (TF) binding motifs makes enhancers the link between the signals that the cells receive and the target transcription factors that regulate gene expression.

Studying enhancers in the process of axis patterning is critical to understand the mechanisms through which the various signaling processes operate. In Hydra the process of axis patterning is also recapitulated during the process of regeneration $[19,29]$. During this process, rapid and large-scale alterations in gene expression take place and these require changes in the chromatin architecture regulated by epigenetic modifications and regulatory regions in the genome [30,31]. Here, we report the conserved characteristics of active histone marks in Hydra which have been described previously in bilaterians. We describe the cis-regulatory elements that exhibit differential H3K27ac modification in response to systemic activation of Wnt signaling. We also show that the nearest neighboring genes of these DNA elements display head specific expression implying their role in head pattern formation. Further, using a specific pharmacological inhibitor of the JMJD3/UTX enzymes that regulate the demethylation of $\mathrm{H} 3 \mathrm{~K} 27 \mathrm{me} 3 / 2$, we demonstrate their role in Wnt signaling mediated phenotypic transformation.

\section{Results}

Genome-wide occupancy profiles of active histone marks in Hydra

As a precedent to understanding the role of differential histone modifications in the oral-aboral axis patterning in Hydra, it is important to characterize the epigenomic landscape of the head organizer. Previous studies have characterized the histone repertoire in Hydra and have identified unique variants such as H2A.X.2 in the gastric region of polyps [32]. In an attempt to understand if there is a unique epigenomic landscape, we sought to characterize the global patterns in histone modifications across the genome of the Hydra adult polyps. Towards this goal, we performed chromatin immunoprecipitation followed by high throughout sequencing (ChIP-seq) for activation-associated histone modifications namely, H3K4me3, H3K27ac and H3K9ac. Correlation analysis of 
the ChIP-seq reads suggests characteristic genomic occupancy specific to each modification (Additional file 1: Fig. $\mathrm{S} 1)$. We investigated their occupancies at gene bodies, putative promoter regions and intergenic regions. Analysis of the gene bodies in the Hydra genome (Fig. 1a and Additional file 2: Table S1) revealed that the occupancy of the activation marks correlates globally with the +1 nucleosome. Further, depletion of the nucleosome represented by low H3 at the TSS marks the nucleosome-free region (NFR) that allows for accessibility of the promoter regions for binding of RNA Pol II also increases the reliability of this analysis [33]. Based on the combinatorial occupancy of these three histone modifications and reads from the transcriptome analysis using RNA-seq, 4 clusters of genes were identified as seen in Fig. 1b. Cluster 1 is characterized by genes that are highly expressed and therefore have very high levels of H3K4me3 across the transcribing gene body and high levels of H3K27ac and H3K9ac close to the TSS to help recruitment of RNA pol II. The genes in cluster 2 exhibit a very low level of expression and relatively low occupancy of the classical activation marks on histone $\mathrm{H} 3$ investigated here. Cluster 3 appears to be genes with no detectable expression but with very low occupancy of both the trimethylation and

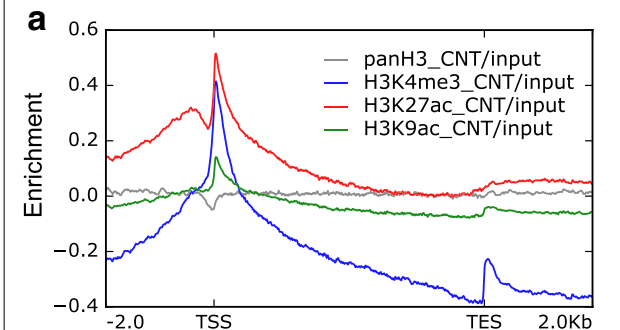

C

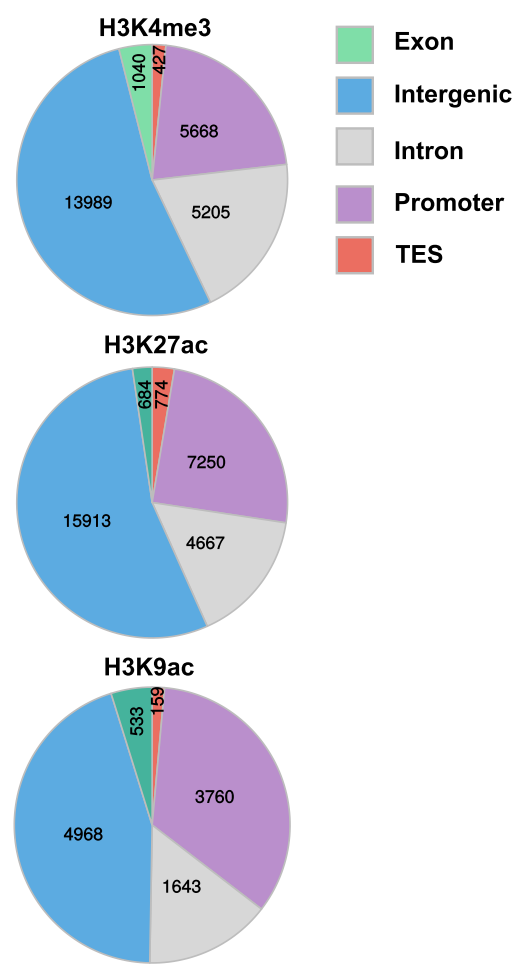

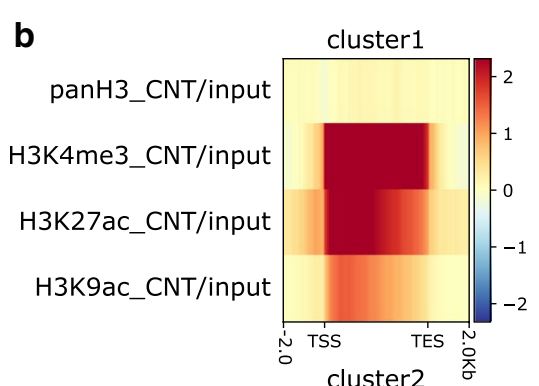
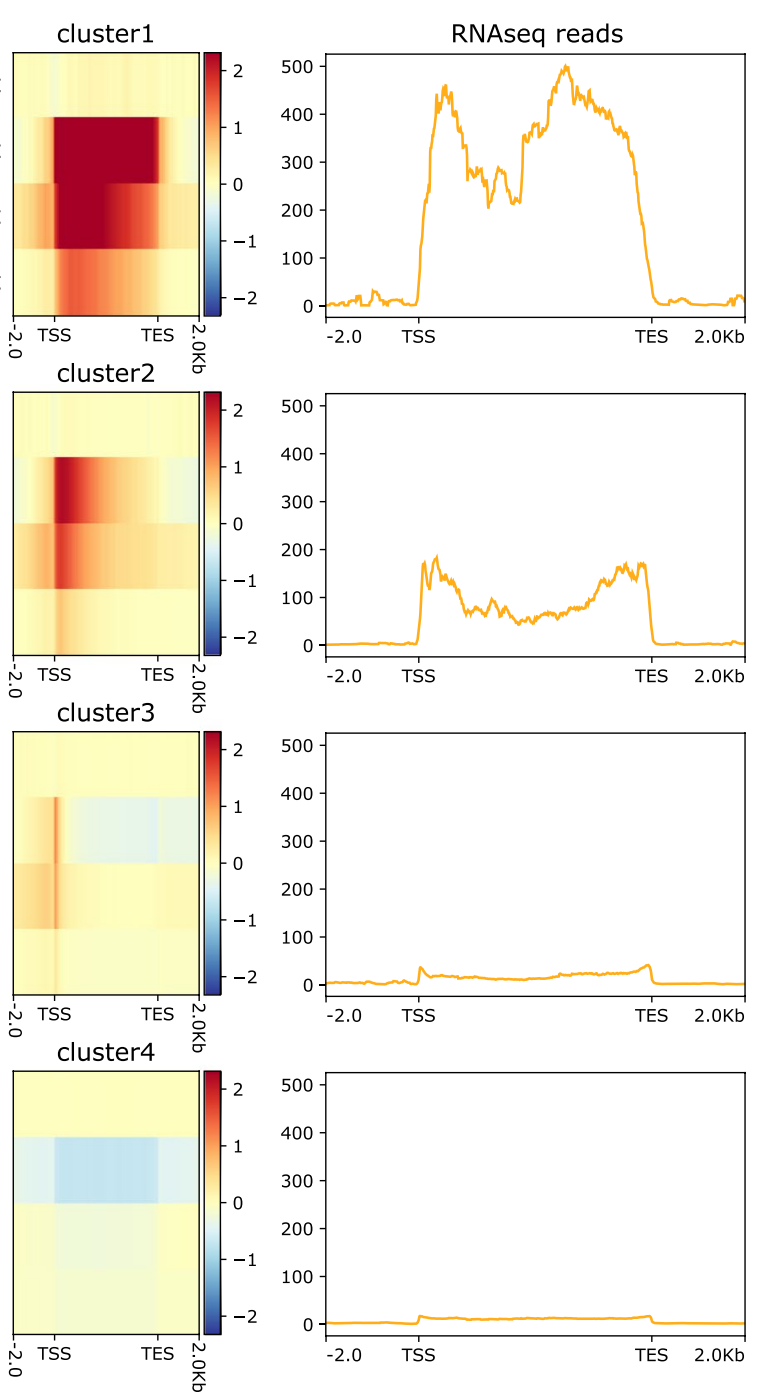

Fig. 1 Global histone modifications across gene bodies in Hydra. a Average profile of the occupancy of the histone H3 and histone modifications H3K4me3, H3K27ac and H3K9ac on genomic regions containing gene bodies (2 Kb upstream and downstream of the transcription start site (TSS) and transcription end site (TES) respectively). $\mathbf{b}$ Heatmaps representing the four k-means clusters (left) and the average profile of the RNA-Seq reads for the clusters (right). c Global occupancy of the three histone modifications H3K4me3, H3K27ac and H3K9ac on different regions of the genome with the numbers representing the number of peaks for the respective histone marks (legend for the colors of the pie-chart is shown on the right) 
the acetylation marks on H3. These genes could be the poised genes and will require the knowledge regarding the presence of histone marks associated with repressive functions to establish their identity. The genes in the 4th cluster are characterized by no detectable histone marks and expression, therefore representing the repressed genes. Further, we sought to characterize the intergenic regions of the Hydra genome based on the occurrence of activating histone modifications. Upon aligning the ChIP-Seq reads to the Hydra magnipapillata genome [34], we obtained the differential occupancy of the three histone marks under investigation across the Hydra genome. The genomic distribution of all the three histone modifications revealed the highest number of peaks at the intergenic regions. Additionally, we observed a higher number of intergenic regions with the occurrence of H3K27ac peaks relative to H3K4me3 peaks (Fig. 1c).

\section{Intergenic regions as enhancers in the Hydra genome}

Differential occupancy of histone modifications is a part of the regulation of gene expression changes caused by structural changes in chromatin. We retrieved the intergenic regions of the Hydra genome excluding the promoters, which displayed the occurrence of H3K27ac as a typical marker for enhancers and investigated the distribution of the three histone modifications namely H3K4me3, H3K27ac and H3K9ac on these intergenic regions. We plotted the occupancy of the three histone modifications on all the intergenic regions and obtained three k-means clusters based on the distribution pattern of the modifications around the peak center (Fig. 2a and Additional file 3: Table S2, Additional file 4: Table S3). Cluster 1 consists of 2884 intergenic regions with a higher level of H3K4me3 than H3K27ac and the distribution of the marks occurs in a broad peak. Cluster 2 consists of 4475 intergenic regions which also exhibits a higher level of H3K4me3 than H3K27ac, however, the distribution of the marks is in sharper peaks. The 8854 regions in cluster 3 have an epigenetic signature where the level of H3K27ac is higher and H3K4me3 is absent. We then plotted the intergenic regions and the average distribution of the histone modifications centering the peaks for each histone mark (Fig. $2 \mathrm{~b}$ and Additional file 3: Table S2). To determine the presence of transcription reads from the intergenic regions, we analyzed the RNA-seq reads originating from these regions. The RNAseq reads in Fig. $2 \mathrm{c}$ depict transcription arising from the corresponding intergenic regions in Fig. $2 \mathrm{a}$ and the 3 clusters shown are representative of the k-means clusters generated based on the distribution of the histone modification. We observed very high levels of transcription arising from the intergenic regions of cluster 1 (Fig. 2c) in addition to the wide peaks of H3K27ac occupancy
(Fig. 2a). Additionally, the occurrence of transcripts from multiple locations across the intergenic DNA elements was also observed as seen in the heat map provided (Fig. 2d). The second cluster obtained also displayed transcription of RNA which was restricted usually to a single originating location and was not spread across the DNA element (Fig. 2c, d). This pattern was similar to the pattern of occupancy of the histone marks on these intergenic regions (Fig. 2a). The third cluster did not show any transcriptional activity and the lack of H3K4me3 mark was striking in this case with a presence of the H3K27ac mark (Fig. 2a, d) which is seen in the regions in cluster 3 of Fig. 2a, c. To understand the role of these intergenic regions in transcriptional regulation, we identified the TFBS motifs present in these regions using the MEMEChIP tool in the MEME Suite package. The motifs across the three clusters which exhibit central enrichment of TFBS in a 500 bp DNA element around the histone modification peak are represented (Fig. 2e, Additional file 1: Fig. S2). Multiple TFBS were identified on the intergenic regions and most of these have a developmental role like the homeodomain-containing TFs, Nkx1-2, Irx3 and PBX3 [93-95]. Notably, we observed significant enrichment of TCF7L2, which is a key target transcription factor of the head patterning $\mathrm{Wnt} / \beta$-catenin signaling pathway in Hydra, on multiple intergenic regions in the 2nd cluster (Fig. 2e, Additional file 1: Fig. S2).

\section{Intergenic genomic DNA is differentially modified for the process of axis patterning in Hydra}

The oral-aboral axis of Hydra is characterized by the molecular and morphological distinction of the body column into the head and foot structures. The canonical Wnt/ $\beta$-catenin signaling pathway plays a critical role in the formation of the head organizer and differentiation of the associated structures such as tentacles and mouth. To understand the molecular mechanisms underlying the head formation and axis patterning in Hydra, we used Alsterpaullone (ALP), a GSK3 $\beta$ inhibitor to induce systemic activation of the $\mathrm{Wnt} / \beta$-catenin signaling pathway in the polyps. Following activation of the Wnt pathway, to decipher the epigenetic regulation of transcription, we performed ChIP-seq for H3K4me3, $\mathrm{H} 3 \mathrm{~K} 27 \mathrm{ac}$ and H3K9ac in both treated and control animals. We then retrieved intergenic regions which show increased occupancy of H3K27ac upon activation of Wnt signaling (Additional file 5: Table S4), which is a classical enhancer associated mark, and monitored the occupancy of H3K4me3 and H3K9ac on these intergenic regions. Based on the differential occupancy of the three marks on the intergenic regions, we obtained four clusters of DNA elements using k-means based clustering (Fig. 3a, Additional file 5: Table S4). Depicted below the 

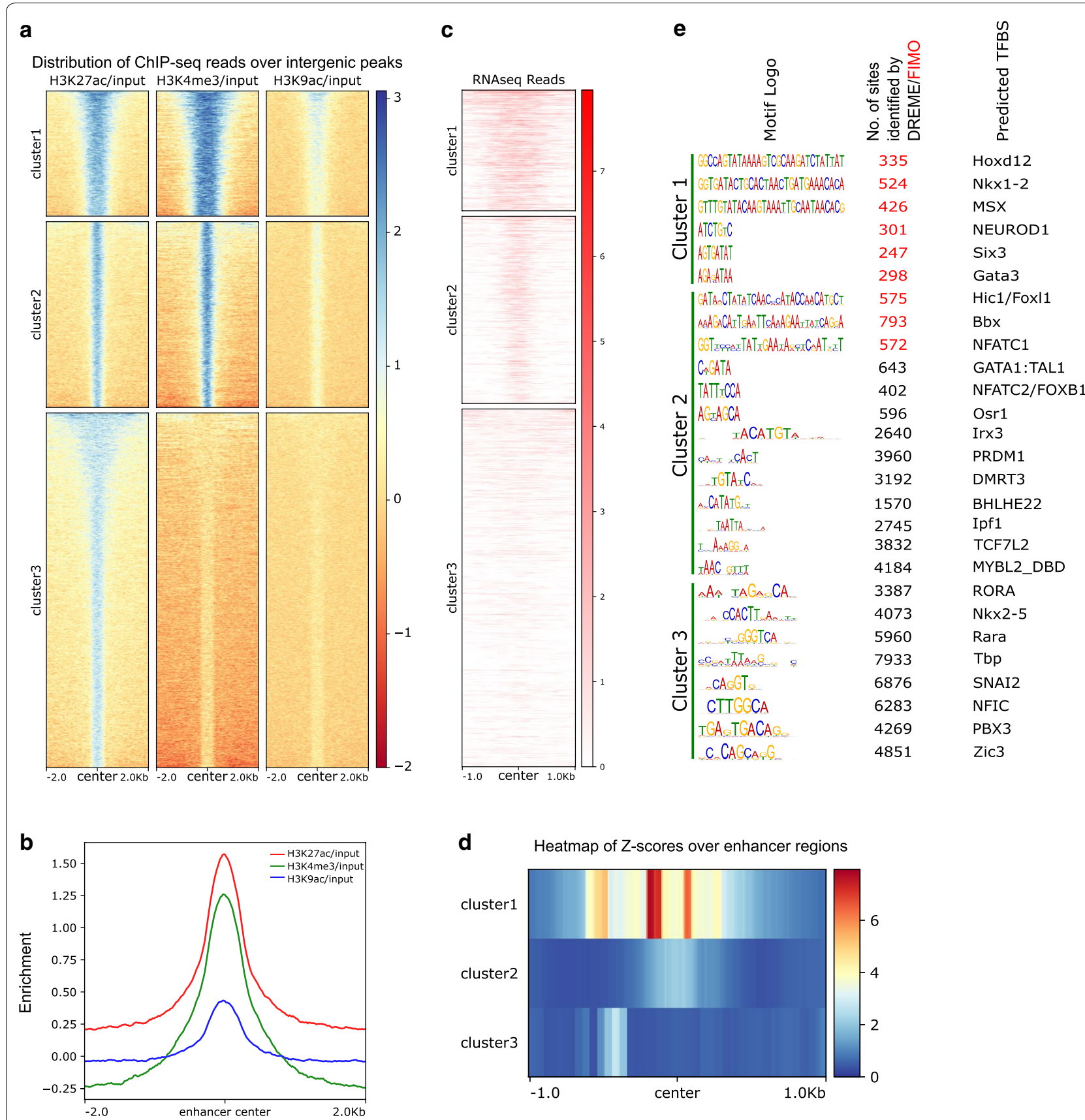

Fig. 2 Enhancer identification and TF motif enrichment in the intergenic regions in Hydra genome. a Heatmap depicting the occupancy of the three histone modifications H3K27ac, H3K4me3 and H3K9ac on the intergenic H3K27ac peaks. Three k-means clusters were obtained based on combinatorial presence of the three modifications. $\mathbf{b}$ The average profile of the modifications around the center of the peak was plotted for all the intergenic regions. c The cluster wise expression of RNA depicted as heatmap based on RNA-Seq reads aligned to the corresponding intergenic regions depicted in ' $\mathbf{a}$ '. $\mathbf{d}$ The Z-scores of the average RNA-seq read profiles for all three clusters over putative enhancer regions. e The transcription factor binding motifs/sites (TFBS) that exhibit central enrichment in the intergenic regions of each cluster. The logos of motifs for the TFBS are shown in the first column and the total number of sites identified by DREME (black font) or FIMO (red font) are shown in the second column. The third column shows the transcription factors identified by TOMTOM [35]

heatmap in Fig. 3a is the average profile of the occurrence of the histone modification in each cluster. The intergenic regions in the clusters 1 and 2, containing
1220 and 601 sequences respectively, display high levels of H3K27ac, H3K4me3 and H3K9ac marks distributed in relatively broad regions surrounding the peak center. 


\section{(See figure on next page.)}

Fig. 3 Dynamic regulation of enhancer-like elements during axis patterning in Hydra. a The occupancy of the H3K27ac, H3K4me3 and H3K9ac on the enriched peak regions identified by activation of Wnt signaling using Alsterpaullone (ALP). Four k-means clusters were obtained based on the combinatorial presence of the three modifications. The average profile of the modifications is depicted below the heatmap of each modification with the colors denoting different clusters. b Distribution of Gene ontology (GO) terms for transcription factors (GO:0003700, GO:0043565) and Wnt signaling pathway members (GO:0016055) for the nearest neighbour genes of the intergenic regions across the four clusters. c Comparison of head specific genes identified by performing differential gene expression analysis using RNA-Seq from head and foot regions (SRR6815024-29) with the nearest neighbour genes of the intergenic regions from clusters 1 \& 2 (cluster1_2) and clusters 3 \& 4 (cluster3_4). d The 774 head specific genes which exhibit enriched H3K27ac mark upon ALP treatment were subjected to network analysis using STRING [38]. Cluster analysis of the network generated showed a large cluster enriched with Wnt signaling pathway members. e The transcription factor binding motifs/sites (TFBS) that show central enrichment in the intergenic H3K27ac peak regions of the 774 genes (intersection of head specific and cluster3_4 genes). The logos of motifs for the TFBS are shown in the first column and the total number of sites identified by DREME (black font) or FIMO (red font) are shown in the second column. The third column shows the transcription factors identified by TOMTOM. f Integrative Genomics Viewer (IGV) [39] track plots depict differential occupancy of H3K27ac and H3K4me3 upon ALP treatment on the gene body and upstream regulatory regions of Hv_Bra 1 which plays a role in head patterning in Hydra. The regions showing enriched H3K27ac peaks are depicted in grey in the last track

There are 6459 intergenic regions belonging to cluster 3 which show occupancy of H3K27ac in sharp peaks and moderate levels of H3K9ac and this cluster shows clear increase in the levels of H3K27ac and H3K9ac upon the ectopic activation of Wnt signaling pathway. The level of H3K4me3 also increases but much more subtly (Fig. 3a). Cluster 4 contains 4729 elements that show an interesting pattern of histone modifications on the intergenic regions. H3K27ac on these intergenic regions is gained upon ALP treatment and H3K9ac levels show a slight reduction in their levels. These regions are depleted of H3K4me3 occupancy following ALP treatment (Fig. 3a). To understand the role these intergenic regions play in transcriptional regulation during axis patterning, we retrieved the genes located nearest to these regions in all 4 clusters. Gene Ontology (GO) analysis enabled the identification of number of transcription factors and Wnt signaling pathway members in all the clusters (Fig. 3b, Additional file 6: Table S5). The clusters 3 and 4 show greater numbers of transcription factors and Wnt signaling pathway members with dynamic regulation via cis-regulatory DNA elements. We compared the genes closest to the intergenic regions in the combined cluster1_2 (clusters 1 \& 2) and the combined cluster3_4 (clusters 3 \& 4) with head specific genes obtained by comparing publicly available RNA-Seq datasets of Hydra head and foot. Here we observed that there are 774 genes that are common between the head and cluster3_4 which correlates with the presence of a larger number of Wnt signaling pathway genes important for head patterning (Fig. 3c, Additional file 1: Fig. S3, Additional file 7: Table S6). Using these 774 genes, a network and clustering analysis was carried out for 237 available orthologs using the STRING database. Following a network enrichment analysis for KEGG pathways, an enrichment of the Wnt and Notch signaling pathway members was observed (Fig. 3d, Additional file 1: Fig. S4, Additional file 8: Table S7). The Wnt signaling pathway was identified as the largest cluster with highly significant interactions and as seen in the network, loci encoding multiple wnt ligands were shown to exhibit differential H3K27ac occupancy on their neighboring intergenic regions. Along with the ligands, neighboring intergenic regions of loci encoding multiple transcription factors such as CnGsc, HyBral, CnASH and CnOtx also display similar differential histone modifications. We performed a motif enrichment analysis using MEME-ChIP on the 774 regions that are common to the head and clusters 3 and 4 and the motifs that show a central enrichment flanking the peak center have been represented in Fig. 3e (Additional file 1: Fig. S5). A majority of the TFs that have been identified belong to the Zinc-coordinating DNA-binding domains superclass of TFs and many of the TFs also have roles in axis patterning, matrix remodeling and nervous system development. Further, multiple TFs that interact with components of the Wnt signaling pathway such as Hic1 and Elk3 were found bind to many intergenic regions [77]. We then investigated the dynamic nature of the histone modifications on few genes that are critical in establishing and maintaining the head organizer activity. Brachyury is a T-box transcription factor that plays a role in tissue patterning during budding and head regeneration in Hydra [36, 37]. Upon constitutive activation of Wnt signaling, Brachyury is upregulated and shows an increase in the occurrence of the histone modifications across the gene body with a greater distribution of the histone modifications proximal to the promoter (Fig. 3f).

\section{Identified cis regulatory elements exhibit enhancer activity} Next, we selected genes that play a critical role head organizer activity with putative enhancer activity and monitored the histone modification profiles of their upstream genomic regions (Fig. 4a). In vitro luciferase assay was performed in HEK293T cells using upstream 


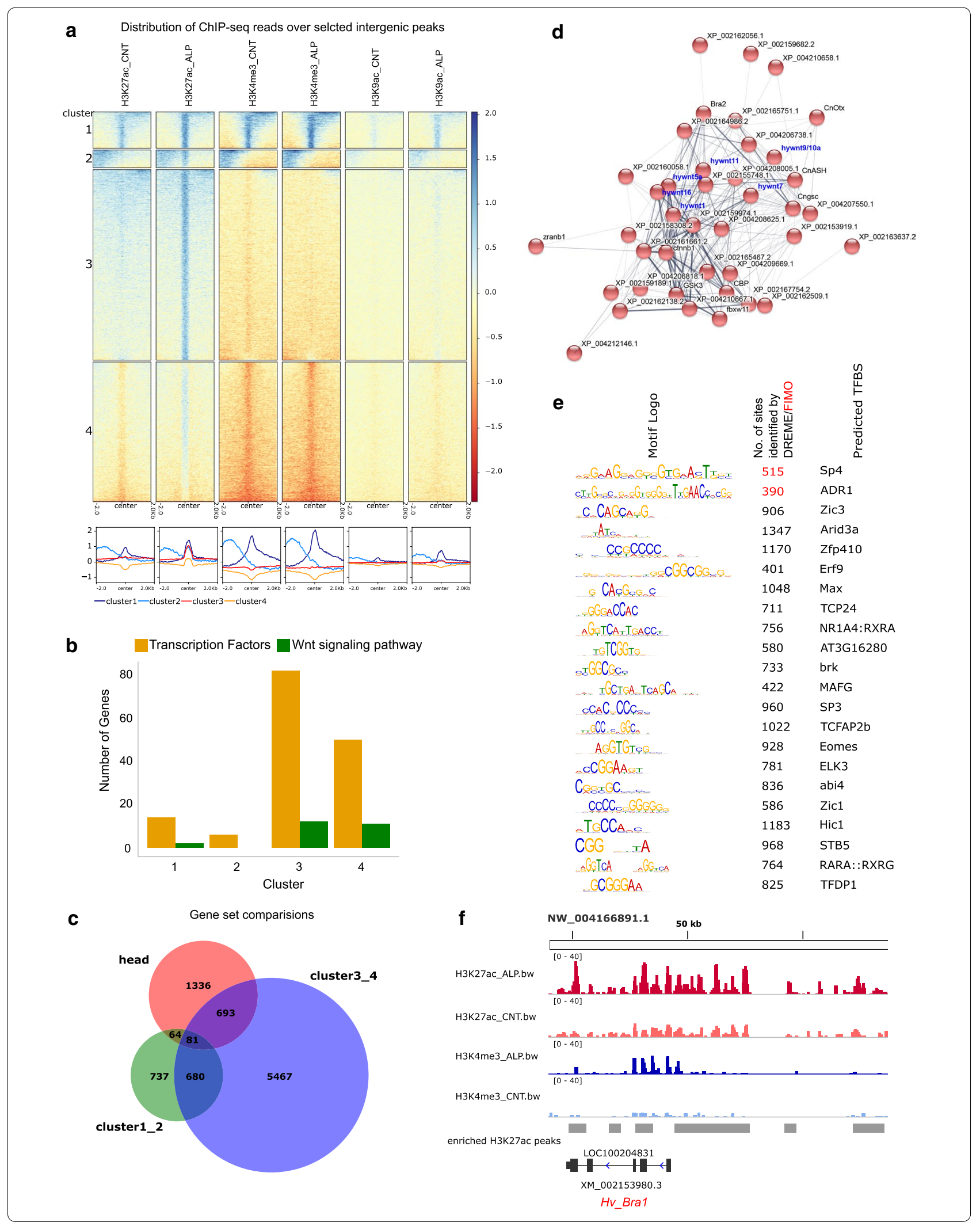


(See figure on next page.)

Fig. 4 Enhancer activity of cis-regulatory elements upstream to multiple Wnts and head organizer genes. Putative enhancer elements were identified based on differential modification of H3K27ac after systemic activation of Wnt signaling and validated by luciferase assay. a Plots of the genomic regions generated using IGV depicting occupancy of the H3K27ac, H3K4me3 histone modifications and RNA expression on distal genomic regions of Hv_Wnt11,Hv_Wnt5a and Hv_Pitx1. ALP-Alsterpaullone treated and VC-vehicle control. The magenta rectangles show the regions cloned into the reporter construct for performing luciferase reporter assays and quantitative ChIP-PCRs. $\mathbf{b}$ The relative luminescence for each of the genomic regions is plotted using pGL3-basic as the control (first column) (** indicates a $p$ value $<0.01$ using Student's t-test). c Relative enrichment of the occupancy of H3K27ac on the neighboring intergenic regions for the target regions has been plotted as fold enrichment over the lgG control in both DMSO controls ( $-5 \mu \mathrm{M} \mathrm{ALP})$ and ALP treated polyps ( $5 \mu \mathrm{M} \mathrm{ALP})\left(^{*}\right.$ indicates a $p$ value $<0.05$ and ${ }^{* *}$ indicates a $p$ value $<0.01$ using Student's t-test)

intergenic (not promoter) regions identified based on H3K27ac modification for genes that are a part of the Wnt signaling network. The upstream genomic regions of Hv_Wnt5a, Hv $v_{-} W n t 7, H v_{-} B r a 1$ and $C n G s c$ which are expressed near the head organizer or tentacles in Hydra exhibited significant enhancer activity (Fig. 4b). Additionally, to validate the enrichment of H3K27ac over these regions in response to systemic activation of Wnt signaling we performed quantitative ChIP-PCR analysis. Here, all the regions could not be tested successfully due to failed amplification during PCR. This could be due to repeated sequences observed in this region. However, except $H v_{-} W n t 7$, the other Wnts $\left(H v_{-} W n t 5 a\right.$ and $H v_{-}$ Wnt11), the head organizer gene Hv_Bral and Hv_Pitx1 exhibit enrichment of H3K27ac (Fig. 4c). This confirms the regulation of these genes by enhancer activity associated with the identified cis-regulatory elements. The differences observed in the luciferase assay and ChIP-PCRs could be due to the variations in the regulatory mechanisms involved under in vivo conditions.

\section{Differential modification of $\mathrm{H} 3 \mathrm{~K} 27$ regulates axis patterning in Hydra}

In Hydra, the canonical Wnt/B-catenin signaling pathway acts as the head organizer and ectopic activation of this pathway is achieved by inhibiting the GSK-3 $\beta$ kinase using Alsterpaullone (ALP). ALP treatment results in multiple molecular and structural changes in the polyp leading to the transformation of the entire body column into a head by giving rise to mis-localized tentacles [40]. As has been previously discussed, such molecular changes involve large-scale global changes in the epigenomic landscape in the cells. To understand the role of specific histone modifications in the axis patterning in Hydra, we used a specific pharmacological inhibitor for KDM6A/6B and activated the Wnt/ $\beta$-catenin signaling pathway using ALP. We observed that when the ALP treated polyps were subjected to the specific inhibitor GSK-J4 that acts against the histone lysine demethylases (KDMs) UTX/JMJD3 (KDM6A/KDM6B) [41], it resulted in a significant reduction in the formation of ectopic tentacles all over the body column of the polyps (Fig. 5a, b,
Additional file 1: Fig. S6). Histone modifications have been shown to occur in a stepwise manner and removal of the trimethylation at H3K27 is essential for the acetylation to occur at the same residue. KDM6 demethylases are implicated in the demethylation of the repressive H3K27me3 mark that enables deposition of the H3K27ac mark which results in the activation of repressed genes $[42,43]$.

\section{Discussion}

The post-genomic era evidenced a shift in focus to noncoding regulatory sequences (cis regulatory elementsCREs) primarily due to their paramount contribution towards the differential regulation of gene regulatory networks (GRNs) comprising conserved gene repertoires in an organism/cell type-specific manner. It is fairly established that CREs play a critical role in the evolution of morphological and cell type diversity [44-47]. Among CREs, enhancer elements in combination with promoter elements control gene expression pattern in spatiotemporal fashion and act as substrates for the evolution of novelties such as morphological and functional diversities $[48,49]$. Most of the studies related to enhancers are limited to selected model organisms. Owing to this, their divergence at the base of metazoans, where major evolutionary transitions took place with respect to developmental processes such as axis pattering and cell type functions, is poorly understood. Until now very few studies have reported the identification of the enhancer elements from the basal metazoan phylum Cnidaria suggesting their roles in gene regulation in different paradigms [31, 50, 51].

Here, we have characterized the histone modification profiles for three histone marks-H3K27ac, H3K9ac and H3K4me3 typically associated with transcriptional activation [52, 53] in Hydra, a member of the phylum Cnidaria. The level and distribution of the histone modifications across the gene body indicates the status of transcription and is different for genes depending on their function [54-56]. Based on the occupancy of the activation-associated histone marks, four clusters of genes have emerged among which the first two represent the 


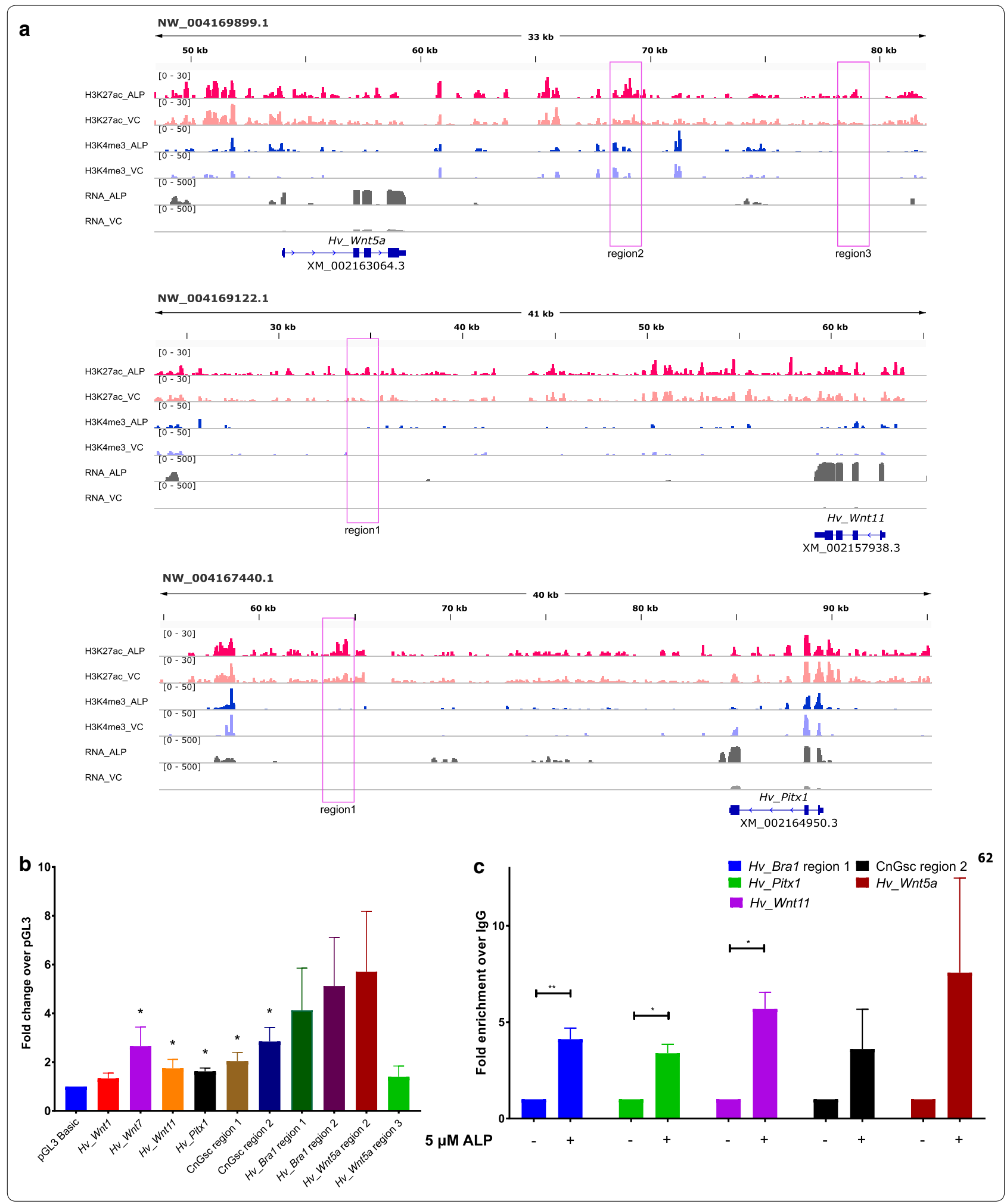

high and intermediate level of expression and a fourth cluster representing the repressed genes. The third cluster presumably depicts a set of poised genes that might be important for the perpetual developmental processes occurring in the Hydra body column [57]. These genes can be activated or repressed based on signaling stimuli 


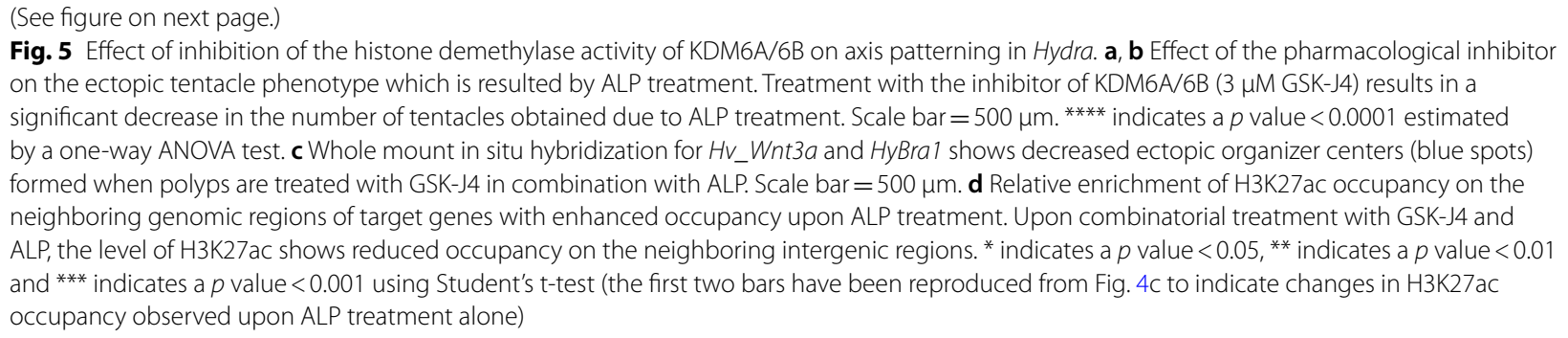

for specific developmental and regenerative processes in a context-dependent manner in Hydra. The occupancy of these histone modifications on a large fraction of the intergenic regions in the genome indicates putative enhancer elements in the Hydra genome. Enhancers are a critical part of the cis regulatory elements for coordinated regulation of gene expression in a signal-dependent and cell-specific manner. Enhancers have been identified and shown to have a conserved mode of function in Nematostella, another Cnidarian [58]. Based on the occupancy of the classical enhancer associated histone mark, H3K27ac, we identified three types of enhancerlike intergenic regions in the Hydra genome. The first cluster exhibits features of super enhancers with broad peaks of H3K27ac, H3K4me3 occupancy and high levels of transcription with multiple originating locations. The second cluster shows the characteristics of typical enhancers with sharp peaks of the activating histone marks and enhancer RNA like transcription events. The third cluster is unique in that it displayed high H3K27ac marks, but no transcription-associated H3K4me3 marks or transcription for the DNA elements. This has been observed in some super enhancers which respond to specific signaling stimuli and do not have an enhancer RNA transcription [59]. We have also identified multiple TFBS on the intergenic regions that belong to TFs involved in development and programming processes in organisms. The TCF7L2 binding site was also identified in the cluster which is the target TF of the head patterning signaling network in Hydra and responds to the $\mathrm{Wnt} / \beta$-catenin signaling stimulus [19].

Upon activation of the canonical Wnt/ $\beta$-catenin signaling pathway in $H y d r a$, we observed differential histone modification on multiple intergenic regions. We identified four clusters based on the differential active histone modifications. Multiple transcription factors and Wnt signaling pathway genes were identified close to the intergenic regions showing changes in their modification status. Among the Wnt signaling pathway members are multiple wnt ligands and many critical transcription factors that play a role in the patterning processes as a response to Wnt signaling [60-65]. The TFBS showing enrichment on the "head-related" intergenic regions have a majority of Zinc finger family of transcription factors which have been known to play roles in patterning processes. Multiple Zic proteins were identified to have TFBS on the Wnt responsive intergenic regions which can bind to multiple transcription factors that play a role in developmental signaling [66-70], chromatin remodeling proteins $[71,72]$ and many nuclear factors. Additionally, the consensus binding motifs for Zic family TFs have been shown to be enriched in enhancers and involves binding to chromatin remodelers [71, 73-76]. Further, we have identified the TF like brk that has a role in transcriptional regulation and the TFs Hic1 and Elk3 that interact with the Wnt signaling pathway to have TFBS in the differentially modified intergenic regions [77].

We have established the enhancer activity for four genomic regions upstream of genes that play a critical role in head patterning namely $H v_{-} W n t 7, H v_{-} W n t 5 a$, CnGsc and Hv_Bra1. Previous studies have shown that the Hv_Wnt11, Hv_Wnt7, Hv_Bral and CnGsc are expressed in the Hydra head organizer [78]. Hv_Wnt5a is localized to epithelial cells which give rise to tentacles during bud development [79] and Hv_Pitx is localized to and important for the organizer formation in the budding zone [80]. This indicates the spatiotemporal regulation of chromatin via H3K27ac and the regulation of these genes during the process of axis determination in an enhancermediated manner. To achieve this type of regulation these genes need to be in relatively close proximity in the genome. However, none of these genes could be located in the same scaffold. This could be presumably due to highly fragmented assemblies of current Hydra genomes available [34]. Chromosome level assembly of Hydra genome will greatly facilitate the studies on cis-regulatory elements.

Further, we have used the H3K27 methylation mark as a paradigm to assess the differential modification of H3K27ac. The change of post-translational modifications on histones from acetylation to methylation has been implicated in regulating gene expression for the processes of epithelial-mesenchymal transition and differentiation in cancers and neuronal progenitors respectively 


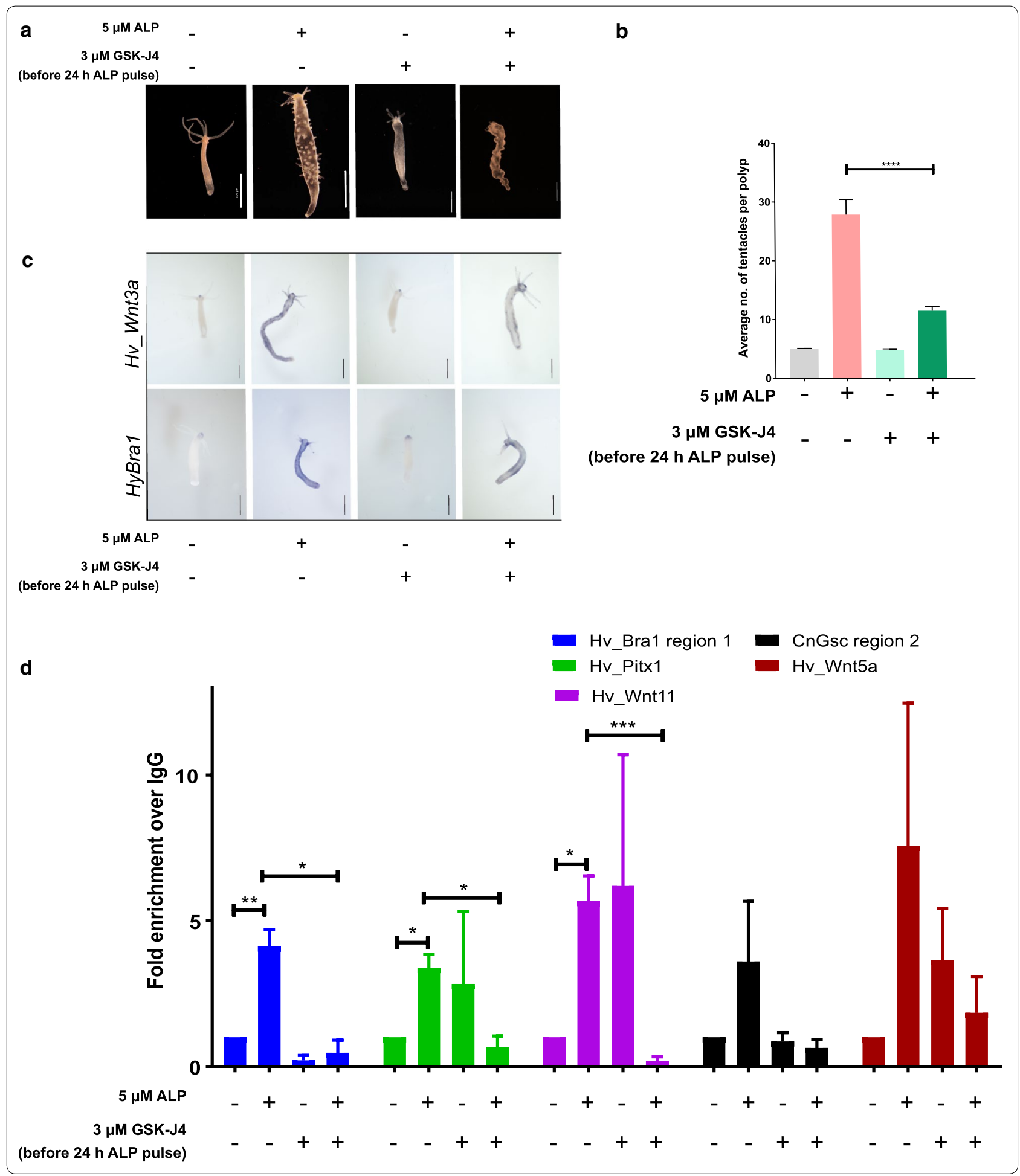

$[81,82]$. We observed genome-wide changes in the H3K27 acetylation status in Hydra upon activation of the Wnt/ $\beta$-catenin signaling pathway which resulted in both the ectopic tentacle phenotype and an expansion of the expression domain of the Wnt target genes like $H v_{-}$Wnt $3 a$ and Hv_Bra1. Disruption of the histone demethylation by pharmacological inhibition of JMJD3/UTX (KDM6A/B) leads to a rescue of the ectopic tentacle 


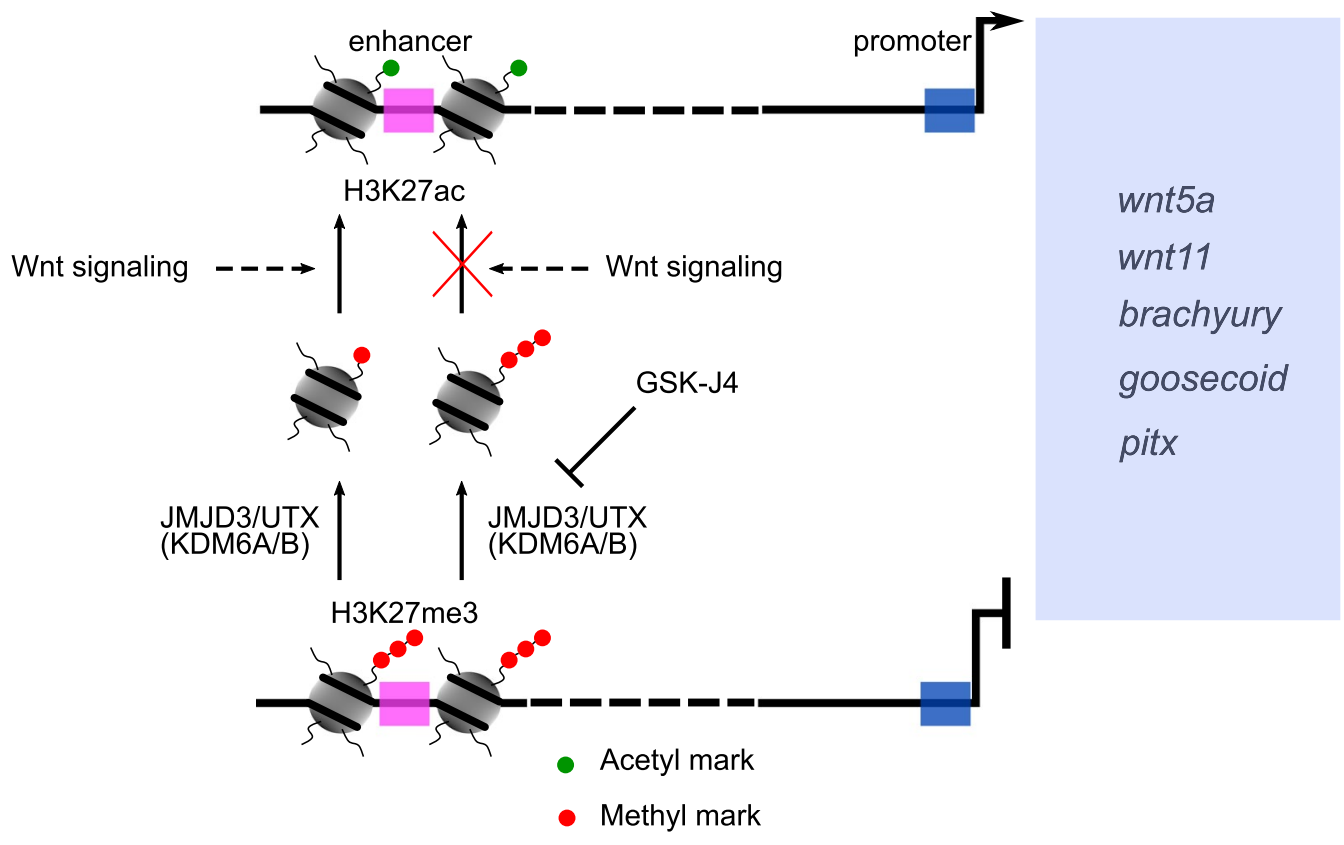

Fig. 6 Graphical summary showing the H3K27ac mediated activation of Hydra homologs of wnt5a, wht11, brachyury, goosecoid and pitx. Wnt signaling causes enrichment of H3K27ac on the enhancer-like elements and it is associated with transcriptional activation. Upon inhibition of the histone demethylase JMJD3/UTX (KDM6A/B), the intergenic cis-regulatory regions cannot be demethylated and are therefore not accessible for deposition of the acetyl mark on the H3K27 residue. This leads to the downregulation of the Wnt target genes and thereby disrupts Wnt directed morphogenesis in Hydra

phenotype caused by the systemic activation of the Wnt/ $\beta$-catenin signaling. This is a result of an altered ability of the H3K27 residue to be acetylated due to disruption in its ability to be demethylated, which transmutes the manifestation of the molecular and morphological changes in Hydra. We propose this H3K27 ac/me switch between the acetylated (ac) and the methylated (me) states as a critical player in the epigenetic regulation of the Wnt/ $\beta$ catenin mediated axis patterning in Hydra (Fig. 6).

\section{Conclusion}

In summary, we have identified the intergenic regulatory elements that possess enhancer-like histone modification signatures such as H3K27Ac and specifically characterized the enhancers that are responsive to Wnt signaling. These putative enhancers are enriched in binding motifs for multiple TFs, among which a majority that belong to the Zinc coordinating domain-containing superfamily with that have been shown to play important roles in developmental processes. Additionally, many TFs known to associate with components of the Wnt signaling pathway such as Hic1, T-box and Ets members have binding motifs on the differentially modified intergenic DNA elements. This investigation also resulted in the identification of candidate pioneer transcription factors that regulate gene expression via cis-regulatory elements in response to Wnt signaling in Hydra. We have demonstrated the in vitro enhancer activity of the cis-regulatory elements for $H v_{-} W n t 7, H v_{-} W n t 5 a, C n G s c$ and $H v_{-}$Bra1. Further, we suggest a role of covalent modification of the histone residue H3K27 as a switch to gain acetylation or methylation and facilitate regulation of the Wnt responsive genes in a spatio-temporal manner.

\section{Methods}

\section{Hydra culture}

A clonal culture of Hydra vulgaris Ind-Pune was maintained at $18{ }^{\circ} \mathrm{C}$ using standard protocols described previously [83, 84]. Hydra polyps were fed with freshly hatched Artemia nauplii larvae daily and cleaned 6-8 h post feeding.

\section{Alsterpaullone treatment}

Hydra polyps were starved for $24 \mathrm{~h}$ and subjected to $5 \mu \mathrm{M}$ Alsterpaullone (ALP) (Sigma-A4847) in hydra medium for $24 \mathrm{~h}$. Control treatments with hydra medium and DMSO as a vehicle control were performed. Alsterpaullone was removed after the pulse and this time point is considered as $0 \mathrm{~h}$ post treatment. The polyps were incubated in hydra medium for an additional $72 \mathrm{~h}$ to observe the morphology of the polyps. 


\section{Inhibitor treatments}

Fifty polyps were used for each of the inhibitor treatments. The chemical inhibitor GSK-J4 (for KDM6A and KDM6B) was obtained from Sigma Aldrich (Cat. No. SML0701). GSK-J4 was used at a final concentration of $3 \mu \mathrm{M}$ to perform all the experiments. The polyps were treated with the demethylase inhibitor for $24 \mathrm{~h}$ before the ALP treatmnetw as started. The demethylase inhibitor was changed every day and the treatment continued for $96 \mathrm{~h}$ while the ALP treatment was performed as previously mentioned. After $96 \mathrm{~h}$, the animals were fixed in $4 \%$ PFA overnight at $4{ }^{\circ} \mathrm{C}$ following relaxation with $2 \%$ urethane. The polyps were stored in 50\% glycerol and imaged. For whole mount in situ hybridization, treatment with GSK-J4 was performed $24 \mathrm{~h}$ before the ALP pulse and the polyps were fixed $24 \mathrm{~h}$ after the ALP pulse.

\section{Chromatin immunoprecipitation (ChIP)}

Two thousand Hydra polyps each for DMSO control and ALP treatment were cross-linked with $1 \%$ formaldehyde, lysed and sonicated in sonication buffer (10 mM Tris$\mathrm{HCl} \mathrm{pH}$ 7.5, $200 \mathrm{mM} \mathrm{NaCl}, 1 \%$ SDS, 4\% NP-40, $1 \mathrm{mM}$ PMSF) to obtain an average chromatin size of $300 \mathrm{bp}$. Chromatin was pre-cleared using $50 \mu \mathrm{l}$ of a $50 \%$ protein A sepharose (GE healthcare) slurry for $1 \mathrm{~h}$ at $4{ }^{\circ} \mathrm{C}$ with gentle inverting. Immunoprecipitations were carried out in $1 \mathrm{ml}$ of ChIP buffer (20 mM Tris- $\mathrm{HCl} \mathrm{pH} \mathrm{8.0,}$ $150 \mathrm{mM} \mathrm{NaCl}, 2 \mathrm{mM}$ EDTA, $1 \%$ Triton-X 100) with AntiH3K4me3, Anti-H3K27ac, Anti-H3K9ac and Anti-H3. An appropriate IgG control was also used with inverting at $4{ }^{\circ} \mathrm{C}$ for $14-16 \mathrm{~h}$. The samples were then incubated with $50 \mu \mathrm{l}$ of a $50 \%$ Protein A sepharose slurry (saturated with $0.5 \%$ BSA and $10 \mathrm{mg} / \mathrm{ml}$ yeast tRNA) for $3 \mathrm{~h}$ at $4{ }^{\circ} \mathrm{C}$ with gentle inverting. ChIP samples were reversecrosslinked, and the DNA was purified using a Qiaquick column (Qiagen). Input chromatin was obtained after preclearing, by de-crosslinking and purifying input DNA using a Qiaquick column (Qiagen) according to manufacturer's instructions. Purified DNA was subjected to library preparation for sequencing or used for quantitative PCR. The fold enrichment was calculated using the formula-Fold enrichment $=2^{\text {(Ct }}$ (Target antibody) $-\mathrm{Ct}(\mathrm{IgG})$ ) The details of the primers used to perform the ChIP RTPCR are provided in Additional file 9: Table S8.

\section{ChIP sequencing}

ChIP-seq was performed using the SOLiD 5500 platform (Applied Biosystems). ChIP-seq libraries were prepared from $5 \mathrm{ng}$ of DNA extracted after chromatin immunoprecipitation for different histone modifications using fragment library construction kit from Life technologies. Here, end repairing of the ChIPed DNA was carried out and then adaptors were ligated using T4 DNA ligase.
Adaptor ligated products were amplified using adaptor specific primers for 12 cycles. As per the manufacturer's protocol DNA purification was performed using Agencourt XP beads (Beckman Coulter). Quality and quantity of the libraries were analyzed using Agilent bioanalyzer 2100 high-sensitivity DNA kit. The pooled libraries were used for sequencing.

\section{ChIP-seq analysis}

Hydra vulgaris reference genome from NCBI was downloaded (GCF_000004095.1_Hydra_RP_1.0) and used for alignment and other analysis [34]. The genome sequence was converted to colour space using bowtie-build and this is used as a reference for alignment of ChIP-seq reads by Bowtie [85]. Using SAMtools [86] aligned sam files were converted to bam files and used for further analysis. Correlation of bam files, peak profiles, heatmaps and cluster analysis were carried out by using deepTools [87]. Identification of ChIP-seq peaks, differential enrichment of peaks and annotation of these peaks were carried out by HOMER [88]. Motif enrichment analysis was performed by extracting 500 bp regions flanking the H3K27ac peak center and subjected to MEME-ChIP using MEME-Suite [35]. Following MEME-ChIP, the obtained motifs were submitted to TOMTOM in MEME-Suite to identify the TF that binds to the intergenic region. RNA-seq data generated previously in the lab [19] was used to show transcription over the selected peak regions (SAMN08966965).

\section{Identification of differentially expressed genes}

Data from NCBI-SRA was downloaded and used for this analysis. Here, sequence reads were aligned to the reference genome using HISAT2 [89]. Aligned bam files were used to get count matrix files using featureCounts function of Subread package [90]. Differential gene expression analysis in head vs foot was carried out by using EdgeR [91].

\section{Luciferase assay}

The selected intergenic regions were cloned into pGL3Basic vector downstream of the luciferase ORF to eliminate chances of promoter-based activity. HEK293T cells were transfected with the target clones along with pRLTK vector expressing Renilla luciferase as a transfection control. The cells were harvested $48 \mathrm{~h}$ post transfection and processed as per the given procedure using the DualLuciferase ${ }^{\circledR}$ Reporter Assay System (Promega). The luminescence was recorded for the target firefly luciferase and Renilla luciferase using EnSight ${ }^{\mathrm{TM}}$ Multimode Microplate 
Reader (PerkinElmer). The firefly luminescence values were normalised to the Renilla luminescence values and the fold change was calculated over the pGL3 basic empty vector normalised values.

\section{Whole mount in situ hybridization}

Digoxigenin-labeled RNA probes for $H v_{-} W n t 3 a$ and $H \nu \_B r a 1$ were prepared by in vitro transcriptions from templates amplified from a recombinant pCR BluntII TOPO (Invitrogen) plasmids containing the $H v_{-}$ Wnt $3 a$ and Hv_Bra1 cDNA using PCR. (DIG Labeling Mix-Sigma-1277073910; SP6 RNA PolymeraseSigma-10810274001; T7 RNA Polymerase-Sigma10881767001). Whole-mount in situ hybridization was performed on the polyps as described [92] with the following changes. Treatment with proteinase- $\mathrm{K}$ was performed for $5 \mathrm{~min}$ and heat-inactivation of the endogenous alkaline phosphatases was done at $70{ }^{\circ} \mathrm{C}$ for $15 \mathrm{~min}$ in 1X SSC. Digoxigenin-labeled RNA probe at a concentration of $150 \mathrm{ng} / \mathrm{ml}$ was used for hybridization at $59^{\circ} \mathrm{C}$. The post-hybridization washes were performed using $1 \mathrm{X}$ SSC-HS gradients. After staining with 50\% NTMT $/ 50 \%$ BM-purple AP substrate for $1 \mathrm{~h}$ at room temperature, the animals were mounted in $80 \%$ glycerol for imaging. Imaging was performed using Olympus MVX10 stereomicroscope.

\section{Supplementary information}

Supplementary information accompanies this paper at https://doi. org/10.1186/s13072-020-00364-6.

\footnotetext{
Additional file 1. Additional figures.

Additional file 2: Table S1. Bed file for locations of ChIP-Seq and RNASeq peaks for genic regions in Hydra.
}

Additional file 3: Table S2. (a) ChIP-Seq peak annotation for $\mathrm{H} 3 \mathrm{~K} 4 \mathrm{me} 3$ in whole Hydra polyps. (b) ChIP-Seq peak annotation for H3K27ac in whole Hydra polyps. (c) ChIP-Seq peak annotation for H3K9ac in whole Hydra polyps.

Additional file 4: Table S3. BED file for H3K27ac ChIP-Seq peaks on intergenic regions.

Additional file 5: Table S4. BED file for H3K27ac ChIP-Seq peaks on intergenic regions enriched after ALP treatment.

Additional file 6: Table S5. (a) Annotated protein list for genes nearest to intergenic regions with H3K27ac enriched after ALP treatment in cluster1. (b) Annotated protein list for genes nearest to intergenic regions with H3K27ac enriched after ALP treatment in cluster2. (c) Annotated protein list for genes nearest to intergenic regions with H3K27ac enriched after ALP treatment in cluster3. (d) Annotated protein list for genes nearest to intergenic regions with H3K27ac enriched after ALP treatment in cluster4.

Additional file 7: Table S6. (a) Gene lists for the four clusters in Fig 3c. (b) List of genes upregulated in the head. (c) List of genes downregulated in the head.

Additional file 8: Table S7. Enrichment of PFAM terms in network analysis. Clustering of the genes identified in the STRING $\mathrm{db}$ and colour codes for the clusters generated.
Additional file 9: Table S8. (a) Primer used for ChIP-qRT-PCR. (b) Relative luminiscence readings for the luciferase assay performed with the target intergenic regions (Source data for Fig. 4b) (c) ddCt values of H3K27ac occupancy estimated using ChIP-RT qPCR (Source data for Fig. 4c) (d) ddCt values of H3K27ac occupancy estimated using ChIP-RT qPCR (Source data for Fig. 5d).

\section{Acknowledgements}

Work was supported by the Centre of Excellence in Epigenetics Program of the Department of Biotechnology, Government of India (BT/01/COE/09/07) to SG. PCR is an Early Career Fellow of the Wellcome-Trust-DBT India Alliance. AG is supported by fellowship from the CSIR. We thank Saurabh J Pradhan for his help with the library preps and sequencing.

\section{Authors' contributions}

PCR, SU and SG conceived the project. AG, PCR and SG wrote the manuscript. PCR, AG, SU performed experiments. PCR performed the ChIP-Seq analysis and transcriptome analysis. PCR, AG and SG interpreted the data. SG supervised the project. All authors read and approved the final manuscript.

\section{Funding}

Work was supported by the Centre of Excellence in Epigenetics Program of the Department of Biotechnology, Government of India (BT/01/COE/09/07) to SG. PCR is an Early Career Fellow of the Wellcome-Trust-DBT India Alliance. AG is supported by fellowship from the CSIR.

\section{Availability of data and materials}

The results of MEME-Suite motif analysis related data are available in Figshare at the https://doi.org/10.6084/m9.figshare.7993853. The ChIP-Seq data is available in NCBI under the following IDs: SRR8893489-97. For the differential gene expression analysis of head versus food transcriptomes, the data was used from the following IDs: (SRR6815024-29). The source data for Fig. 4b, c and 5d is available in Additional file 9: Table 58.

Ethics approval and consent to participate

Not applicable.

\section{Consent for publication}

Not applicable.

\section{Competing interests}

The authors declare that they have no competing interests.

Received: 30 May 2020 Accepted: 26 September 2020

Published online: 12 October 2020

\section{References}

1. Nusslein-Volhard C, Wieschaus E. Mutations affecting segment number and polarity in Drosophila. Nature. 1980;287(5785):795-801.

2. Niehrs C. Regionally specific induction by the Spemann-Mangold organizer. Nat Rev Genet. 2004;5(6):425

3. Slack JM, Holland PW, Graham CF. The zootype and the phylotypic stage. Nature. 1993;361(6412):490-2.

4. Schummer M, Scheurlen I, Schaller C, Galliot B. HOM/HOX homeobox genes are present in hydra (Chlorohydra viridissima) and are differentially expressed during regeneration. EMBO J. 1992;11(5):1815-23.

5. Shenk MA, Bode HR, Steele RE. Expression of Cnox-2, a HOM/HOX homeobox gene in hydra, is correlated with axial pattern formation. Development. 1993;117(2):657-67.

6. Shenk MA, Gee L, Steele RE, Bode HR. Expression of Cnox-2, a HOM/ HOX gene, is suppressed during head formation in hydra. Dev Biol. 1993;160(1):108-18.

7. Gauchat D, Mazet F, Berney C, Schummer M, Kreger S, Pawlowski J, et al. Evolution of Antp-class genes and differential expression of Hydra Hox/paraHox genes in anterior patterning. Proc Natl Acad Sci USA. 2000;97(9):4493-8. 
8. Kamm K, Schierwater B. Ancient complexity of the non-Hox ANTP gene complement in the anthozoan Nematostella vectensis: implications for the evolution of the ANTP superclass. J Exp Zool B Mol Dev Evol. 2006;306(6):589-96.

9. Ryan JF, Mazza ME, Pang K, Matus DQ, Baxevanis AD, Martindale MQ, et al. Pre-bilaterian origins of the Hox cluster and the Hox code: evidence from the sea anemone, Nematostella vectensis. PLoS ONE. 2007;2(1):e153.

10. Reddy PC, Unni MK, Gungi A, Agarwal P, Galande S. Evolution of Hox-like genes in Cnidaria: study of Hydra Hox repertoire reveals tailor-made Hoxcode for Cnidarians. Mech Dev. 2015;138(Pt 2):87-96.

11. He S, Del Viso F, Chen CY, Ikmi A, Kroesen AE, Gibson MC. An axial Hox code controls tissue segmentation and body patterning in Nematostella vectensis. Science. 2018;361(6409):1377-80.

12. Petersen CP, Reddien PW. Wnt signaling and the polarity of the primary body axis. Cell. 2009;139(6):1056-68.

13. Lee PN, Pang K, Matus DQ, Martindale MQ. A WNT of things to come: evolution of Wnt signaling and polarity in cnidarians. Semin Cell Dev Biol. 2006;17(2):157-67.

14. Finnerty JR, Master VA, Irvine S, Kourakis MJ, Warriner S, Martindale MQ. Homeobox genes in the Ctenophora: identification of paired-type and Hox homologues in the atentaculate ctenophore, Beroe ovata. Mol Mar Biol Biotechnol. 1996;5(4):249-58.

15. Gufler S, Artes B, Bielen H, Krainer I, Eder MK, Falschlunger J, et al. betaCatenin acts in a position-independent regeneration response in the simple eumetazoan Hydra. Dev Biol. 2018;433(2):310-23.

16. Hobmayer B, Rentzsch F, Kuhn K, Happel CM, von Laue CC, Snyder P, et al. WNT signalling molecules act in axis formation in the diploblastic metazoan Hydra. Nature. 2000;407(6801):186-9.

17. Plickert G, Jacoby V, Frank U, Muller WA, Mokady O. Wnt signaling in hydroid development: formation of the primary body axis in embryogenesis and its subsequent patterning. Dev Biol. 2006;298(2):368-78.

18. Wikramanayake AH, Hong M, Lee PN, Pang K, Byrum CA, Bince JM, et al. An ancient role for nuclear beta-catenin in the evolution of axial polarity and germ layer segregation. Nature. 2003;426(6965):446-50.

19. Reddy PC, Gungi A, Ubhe S, Pradhan SJ, Kolte A, Galande S. Molecular signature of an ancient organizer regulated by Wnt/beta-catenin signalling during primary body axis patterning in Hydra. Commun Biol. 2019;2:434

20. Maston GA, Evans SK, Green MR. Transcriptional regulatory elements in the human genome. Annu Rev Genomics Hum Genet. 2006;7:29-59.

21. Arnold CD, Gerlach D, Stelzer C, Boryn LM, Rath M, Stark A. Genome-wide quantitative enhancer activity maps identified by STARR-seq. Science. 2013:339(6123):1074-7.

22. Bonn S, Zinzen RP, Girardot C, Gustafson EH, Perez-Gonzalez A, Delhomme $\mathrm{N}$, et al. Tissue-specific analysis of chromatin state identifies temporal signatures of enhancer activity during embryonic development. Nat Genet. 2012;44(2):148-56.

23. Heintzman ND, Hon GC, Hawkins RD, Kheradpour P, Stark A, Harp LF, et al. Histone modifications at human enhancers reflect global cell-typespecific gene expression. Nature. 2009;459(7243):108-12.

24. Pekowska A, BenoukrafT, Zacarias-Cabeza J, Belhocine M, Koch F, Holota $\mathrm{H}$, et al. H3K4 tri-methylation provides an epigenetic signature of active enhancers. EMBO J. 2011;30(20):4198-210.

25. Fang $Y$, Wang $Y$, Zhu Q, Wang J, Li G. In silico identification of enhancers on the basis of a combination of transcription factor binding motif occurrences. Sci Rep. 2016:6:32476.

26. Djebali S, Davis CA, Merkel A, Dobin A, Lassmann T, Mortazavi A, et al. Landscape of transcription in human cells. Nature. 2012;489(7414):101-8.

27. Heintzman ND, Stuart RK, Hon G, Fu Y, Ching CW, Hawkins RD, et al. Distinct and predictive chromatin signatures of transcriptional promoters and enhancers in the human genome. Nat Genet. 2007;39(3):311-8.

28. Andersson R, Sandelin A, Danko CG. A unified architecture of transcriptional regulatory elements. Trends Genet. 2015;31(8):426-33.

29. Wenger Y, Buzgariu W, Perruchoud C, Loichot G, Galliot B. Generic and context-dependent gene modulations during Hydra whole body regeneration. bioRxiv. 2019:587147. https://doi.org/10.1101/587147.

30. Petersen HO, Hoger SK, Looso M, Lengfeld T, Kuhn A, Warnken U, et al. A comprehensive transcriptomic and proteomic analysis of hydra head regeneration. Mol Biol Evol. 2015;32(8):1928-47.

31. Murad R, Macias-Muñoz A, Wong A, Ma X, Mortazavi A. Integrative analysis of Hydra head regeneration reveals activation of distal enhancer-like elements. bioRxiv. 2019:544049. https://doi.org/10.1101/544049.
32. Reddy PC, Ubhe S, Sirwani N, Lohokare R, Galande S. Rapid divergence of histones in Hydrozoa (Cnidaria) and evolution of a novel histone involved in DNA damage response in hydra. Zoology. 2017;123:53-63.

33. Radman-Livaja M, Rando OJ. Nucleosome positioning: how is it established, and why does it matter? Dev Biol. 2010;339(2):258-66.

34. Chapman JA, Kirkness EF, Simakov O, Hampson SE, Mitros T, Weinmaier T, et al. The dynamic genome of Hydra. Nature. 2010;464(7288):592-6.

35. Bailey TL, Boden M, Buske FA, Frith M, Grant CE, Clementi L, et al. MEME SUITE: tools for motif discovery and searching. Nucleic Acids Res. 2009;37(Web Server issue):W202-W208208.

36. Bielen H, Oberleitner S, Marcellini S, Gee L, Lemaire P, Bode HR, et al. Divergent functions of two ancient Hydra Brachyury paralogues suggest specific roles for their C-terminal domains in tissue fate induction. Development. 2007;134(23):4187-97.

37. Technau U, Bode HR. HyBra1, a Brachyury homologue, acts during head formation in Hydra. Development. 1999;126(5):999-1010.

38. Szklarczyk D, Gable AL, Lyon D, Junge A, Wyder S, Huerta-Cepas J, et al. STRING v11: protein-protein association networks with increased coverage, supporting functional discovery in genome-wide experimental datasets. Nucleic Acids Res. 2019:47(D1):D607-D613613.

39. Robinson JT, Thorvaldsdottir H, Winckler W, Guttman M, Lander ES, Getz $\mathrm{G}$, et al. Integrative genomics viewer. Nat Biotechnol. 2011;29(1):24-6.

40. Broun M, Gee L, Reinhardt B, Bode HR. Formation of the head organizer in hydra involves the canonical Wnt pathway. Development. 2005;132(12):2907-16.

41. Kruidenier L, Chung CW, Cheng Z, Liddle J, Che K, Joberty G, et al. A selective jumonji H3K27 demethylase inhibitor modulates the proinflammatory macrophage response. Nature. 2012;488(7411):404-8.

42. Petruk S, Black KL, Kovermann SK, Brock HW, Mazo A. Stepwise histone modifications are mediated by multiple enzymes that rapidly associate with nascent DNA during replication. Nat Commun. 2013:4:2841.

43. Wang W, Lim KG, Feng M, Bao Y, Lee PL, Cai Y, et al. KDM6B counteracts EZH2-mediated suppression of IGFBP5 to confer resistance to PI3K/AKT inhibitor treatment in breast cancer. Mol Cancer Ther. 2018;17(9):1973-83.

44. Carroll SB. Evo-devo and an expanding evolutionary synthesis: a genetic theory of morphological evolution. Cell. 2008;134(1):25-36.

45. Stern DL, Orgogozo V. The loci of evolution: how predictable is genetic evolution? Evolution. 2008;62(9):2155-77.

46. Wittkopp PJ, Kalay G. Cis-regulatory elements: molecular mechanisms and evolutionary processes underlying divergence. Nat Rev Genet. 2012;13(1):59-69.

47. Wray GA. The evolutionary significance of cis-regulatory mutations. Nat Rev Genet. 2007;8(3):206-16.

48. Levine M. Transcriptional enhancers in animal development and evolution. Curr Biol. 2010;20(17):R754-R763763.

49. Ong C-T, Corces VG. Enhancer function: new insights into the regulation of tissue-specific gene expression. Nat Rev Genet. 2011;12(4):283-93.

50. Schwaiger M, Schönauer A, Rendeiro AF, Pribitzer C, Schauer A, Gilles $A F$, et al. Evolutionary conservation of the eumetazoan gene regulatory landscape. Genome Res. 2014;24(4):639-50.

51. Weizman EN, Tannenbaum M, Tarrant AM, Hakim O, Levy O. Chromatin dynamics enable transcriptional rhythms in the cnidarian Nematostella vectensis. PLoS Genet. 2019;15(11):e1008397.

52. Creyghton MP, Cheng AW, Welstead GG, Kooistra T, Carey BW, Steine EJ, et al. Histone H3K27ac separates active from poised enhancers and predicts developmental state. Proc Natl Acad Sci USA. 2010;107(50):21931-6.

53. Wang Z, Zang C, Rosenfeld JA, Schones DE, Barski A, Cuddapah S, et al. Combinatorial patterns of histone acetylations and methylations in the human genome. Nat Genet. 2008;40(7):897.

54. Chen K, Chen Z, Wu D, Zhang L, Lin X, Su J, et al. Broad H3K4me3 is associated with increased transcription elongation and enhancer activity at tumor-suppressor genes. Nat Genet. 2015:47(10):1149-57.

55. Okitsu CY, Hsieh JC, Hsieh CL. Transcriptional activity affects the $\mathrm{H} 3 \mathrm{~K} 4 \mathrm{me} 3$ level and distribution in the coding region. Mol Cell Biol. 2010;30(12):2933-46

56. Pu M, Wang M, Wang W, Velayudhan SS, Lee SS. Unique patterns of trimethylation of histone $\mathrm{H} 3$ lysine 4 are prone to changes during aging in Caenorhabditis elegans somatic cells. PLoS Genet. 2018;14(6):e1007466.

57. Muller WA. Pattern formation in the immortal Hydra. Trends Genet. 1996;12(3):91-6. 
58. Schwaiger M, Schonauer A, Rendeiro AF, Pribitzer C, Schauer A, Gilles $A F$, et al. Evolutionary conservation of the eumetazoan gene regulatory landscape. Genome Res. 2014;24(4):639-50.

59. Hah N, Benner C, Chong LW, Yu RT, Downes M, Evans RM. Inflammationsensitive super enhancers form domains of coordinately regulated enhancer RNAs. Proc Natl Acad Sci USA. 2015;112(3):E297-302.

60. Zhang Z, Deb A, Zhang Z, Pachori A, He W, Guo J, et al. Secreted frizzled related protein 2 protects cells from apoptosis by blocking the effect of canonical Wnt3a. J Mol Cell Cardiol. 2009;46(3):370-7.

61. Arnold SJ, Stappert J, Bauer A, Kispert A, Herrmann BG, Kemler R. Brachyury is a target gene of the Wnt/beta-catenin signaling pathway. Mech Dev. 2000;91(1-2):249-58.

62. Niehrs C, Steinbeisser H, De Robertis EM. Mesodermal patterning by a gradient of the vertebrate homeobox gene goosecoid. Science. 1994;263(5148):817-20

63. Puelles E, Acampora D, Lacroix E, Signore M, Annino A, Tuorto F, et al. Otx dose-dependent integrated control of antero-posterior and dorso-ventral patterning of midbrain. Nat Neurosci. 2003;6(5):453-60.

64. Wheeler SR, Carrico ML, Wilson BA, Brown SJ, Skeath JB. The expression and function of the achaete-scute genes in Tribolium castaneum reveals conservation and variation in neural pattern formation and cell fate specification. Development. 2003;130(18):4373-81.

65. Lolas M, Valenzuela PD, Tjian R, Liu Z. Charting Brachyury-mediated developmental pathways during early mouse embryogenesis. Proc Natl Acad Sci USA. 2014;111(12):4478-83.

66. Fujimi TJ, Hatayama M, Aruga J. Xenopus Zic3 controls notochord and organizer development through suppression of the Wnt/beta-catenin signaling pathway. Dev Biol. 2012;361(2):220-31.

67. Koyabu Y, Nakata K, Mizugishi K, Aruga J, Mikoshiba K. Physical and functional interactions between Zic and Gli proteins. J Biol Chem. 2001;276(10):6889-922.

68. Pardo M, Lang B, Yu L, Prosser H, Bradley A, Babu MM, et al. An expanded Oct4 interaction network: implications for stem cell biology, development, and disease. Cell Stem Cell. 2010;6(4):382-95.

69. Pourebrahim R, Houtmeyers R, Ghogomu S, Janssens S, Thelie A, Tran HT, et al. Transcription factor Zic2 inhibits Wnt/beta-catenin protein signaling. J Biol Chem. 2011;286(43):37732-40.

70. Sanchez-Ferras O, Bernas G, Laberge-Perrault E, Pilon N. Induction and dorsal restriction of Paired-box 3 (Pax3) gene expression in the caudal neuroectoderm is mediated by integration of multiple pathways on a short neural crest enhancer. Biochim Biophys Acta. 2014;1839(7):546-58.

71. Luo Z, Gao X, Lin C, Smith ER, Marshall SA, Swanson SK, et al. Zic2 is an enhancer-binding factor required for embryonic stem cell specification. Mol Cell. 2015;57(4):685-94.

72. Zhu P, Wang Y, He L, Huang G, Du Y, Zhang G, et al. ZIC2-dependent OCT4 activation drives self-renewal of human liver cancer stem cells. J Clin Investig. 2015;125(10):3795-808.

73. Sankar S, Yellajoshyula D, Zhang B, Teets B, Rockweiler N, Kroll KL. Gene regulatory networks in neural cell fate acquisition from genome-wide chromatin association of Geminin and Zic1. Sci Rep. 2016;6:37412.

74. Simoes-Costa MS, McKeown SJ, Tan-Cabugao J, Sauka-Spengler T, Bronner ME. Dynamic and differential regulation of stem cell factor FoxD3 in the neural crest is encrypted in the genome. PLoS Genet. 2012;8(12):e1003142.

75. Winata CL, Kondrychyn I, Kumar V, Srinivasan KG, Orlov Y, Ravishankar A, et al. Genome wide analysis reveals Zic3 interaction with distal regulatory elements of stage specific developmental genes in zebrafish. PLoS Genet. 2013;9(10):e1003852.

76. Yu W, Briones V, Lister R, McIntosh C, Han Y, Lee EY, et al. CG hypomethylation in Lsh-/- mouse embryonic fibroblasts is associated with de novo H3K4me1 formation and altered cellular plasticity. Proc Natl Acad Sci USA. 2014;111(16):5890-5.

77. Valenta T, Lukas J, Doubravska L, Fafilek B, Korinek V. HIC1 attenuates Wnt signaling by recruitment of TCF-4 and beta-catenin to the nuclear bodies. EMBO J. 2006;25(11):2326-37.
78. Lengfeld T, Watanabe H, Simakov O, Lindgens D, Gee L, Law L, et al. Multiple Wnts are involved in Hydra organizer formation and regeneration. Dev Biol. 2009;330(1):186-99.

79. Philipp I, Aufschnaiter R, Ozbek S, Pontasch S, Jenewein M, Watanabe H, et al. Wnt/beta-catenin and noncanonical Wnt signaling interact in tissue evagination in the simple eumetazoan Hydra. Proc Natl Acad Sci USA. 2009:106(11):4290-5.

80. Watanabe H, Schmidt HA, Kuhn A, Hoger SK, Kocagoz Y, Laumann-Lipp $\mathrm{N}$, et al. Nodal signalling determines biradial asymmetry in Hydra. Nature. 2014;515(7525):112-5.

81. Song Y, Wang R, Li LW, Liu X, Wang YF, Wang QX, et al. Long non-coding RNA HOTAIR mediates the switching of histone $\mathrm{H} 3$ lysine 27 acetylation to methylation to promote epithelial-to-mesenchymal transition in gastric cancer. Int J Oncol. 2019;54(1):77-86.

82. Balmer NV, Klima S, Rempel E, Ivanova VN, Kolde R, Weng MK, et al. From transient transcriptome responses to disturbed neurodevelopment: role of histone acetylation and methylation as epigenetic switch between reversible and irreversible drug effects. Arch Toxicol. 2014;88(7):1451-68.

83. Reddy PC, Barve A, Ghaskadbi S. Description and phylogenetic characterization of common hydra from India. Curr Sci. 2011;101(6):736-8.

84. Horibata Y, Sakaguchi K, Okino N, lida H, Inagaki M, Fujisawa T, et al. Unique catabolic pathway of glycosphingolipids in a hydrozoan, Hydra magnipapillata, involving endoglycoceramidase. J Biol Chem. 2004;279(32):33379-89.

85. Langmead B. Aligning short sequencing reads with Bowtie. Curr Protoc Bioinform. 2010; Chapter 11:Unit 117.

86. Li H, Handsaker B, Wysoker A, Fennell T, Ruan J, Homer N, et al. The Sequence Alignment/Map format and SAMtools. Bioinformatics. 2009:25(16):2078-9.

87. Ramirez F, Dundar F, Diehl S, Gruning BA, Manke T. deepTools: a flexible platform for exploring deep-sequencing data. Nucleic Acids Res. 2014;42(Web Server issue):W187-W191191.

88. Heinz S, Benner C, Spann N, Bertolino E, Lin YC, Laslo P, et al. Simple combinations of lineage-determining transcription factors prime cisregulatory elements required for macrophage and B cell identities. Mol Cell. 2010;38(4):576-89.

89. Kim D, Langmead B, Salzberg SL. HISAT: a fast spliced aligner with low memory requirements. Nat Methods. 2015;12(4):357-60.

90. Liao Y, Smyth GK, Shi W. The Subread aligner: fast, accurate and scalable read mapping by seed-and-vote. Nucleic Acids Res. 2013;41(10):e108.

91. Robinson MD, McCarthy DJ, Smyth GK. edgeR: a Bioconductor package for differential expression analysis of digital gene expression data. Bioinformatics. 2010;26(1):139-40.

92. Martinez DE, Dirksen ML, Bode PM, Jamrich M, Steele RE, Bode HR. Budhead, a fork head/HNF-3 homologue, is expressed during axis formation and head specification in hydra. Dev Biol. 1997;192(2):523-36.

93. Selleri L, Zappavigna V, Ferretti E. 'Building a perfect body': control of vertebrate organogenesis by PBX-dependent regulatory networks. Genes Dev. 2019:33(5-6):258-75.

94. Dana AA, Tamashiro VB, Alarcon YM. Nkx1-2 is a transcriptional repressor and is essential for the activation of Brachyury in P19 mouse embryonal carcinoma cell. Differentiation. 2012;83(5):282-92.

95. Rodríguez-Seguel E, Alarcón P, Gómez-Skarmeta JL. The Xenopus Irx genes are essential for neural patterning and define the border between prethalamus and thalamus through mutual antagonism with the anterior repressors Fezf and Arx. Dev Biol. 2009;329(2):258-68.

\section{Publisher's Note}

Springer Nature remains neutral with regard to jurisdictional claims in published maps and institutional affiliations. 